\title{
Cancer du sein : optimisation du parcours de soins Congrès de la Société Française de Sénologie et de Pathologie Mammaire (SFSPM) 2018
}

\author{
Breast Cancer: Optimisation of the Care Process
}

\author{
D. Serin \\ (C) Lavoisier SAS 2019
}

\section{Chère lectrice,}

Cher lecteur d'Oncologie,

Vous tenez entre vos mains le numéro d'Oncologie consacré aux débats qui ont animé les $40^{\mathrm{es}}$ Journées de la Société française de sénologie et de pathologie mammaire qui se sont tenues à Avignon en novembre 2018.

Nous avions choisi pour ce congrès national un thème d'actualité : Cancer du sein : optimisation du parcours de soins.

Ce sujet, très transversal, plus organisationnel que scientifique, plus santé publique que médical, a rencontré un très vif succès, et plus de 1200 personnes ont fréquenté les salles du Palais des Papes. Ce bâtiment chargé d'histoire où se sont tenus tant de conciles historiques était sûrement le lieu le plus indiqué en France pour inviter tous les acteurs impliqués dans ce domaine à partager leurs expériences, leurs difficultés mais aussi leurs réalisations et leurs espoirs pour améliorer cette traversée de la maladie. Nous avons fait une large place aux représentantes des malades et de leurs proches, que ce soit la Ligue 84 de lutte contre le cancer, Europa Donna, Vivre Comme Avant.

La pluridisciplinarité chère à la SFSPM s'est exprimée au travers des prises de parole des intervenants médicaux venant de toutes les spécialités concernées, mais nous avons élargi notre vision grâce à la parole issue d'autres métiers, d'autres responsables engagés dans l'optimisation de ce parcours de soins : HAS, ARS, Inca, Unicancer, hospitalisation privée Elsan, infirmière de pratique avancée, sociologue, et l'expérience européenne d'EUSOMA.

Vous trouverez sur le site de la SFSPM (www.senologie. com) outre le programme un enregistrement de toutes les présentations faites en session plénière.

Alors pourquoi ce numéro d'Oncologie « spécial SFSPM — Avignon $2018 » ?$

\section{Serin $(\bowtie)$}

Institut Sainte-Catherine, 250, chemin de Baigne-Pieds, CS 80005, F-84918 Avignon cedex 09, France e-mail : d.serin@isc84.org
Tout d'abord parce que je crois encore à la vertu du papier. Garder une revue dans notre bibliothèque, la feuilleter, la relire, cela reste un vrai plaisir même si ce n'est plus une nécessité grâce à l'informatique.

En deuxième lieu et connaissant l'exhaustivité du site Internet de la SFSPM, je souhaitais conserver une trace écrite des efforts de rédaction qu'ont développé tous les intervenants d'Avignon. La longueur des textes qu'ils nous ont fait parvenir est variable et a été volontairement libérée de toute contrainte. Ce ne sont pas des articles répondant à des critères académiques ou éditologiques classiques, ils correspondent à l'expression du cœur de leur présentation orale, et nous les remercions d'avoir accepté ce choix éditologique inhabituel.

Enfin, dans le discours d'ouverture du congrès de novembre 2018, nous nous demandions (en ayant une petite idée derrière la tête) si le parcours de soins allait devenir un élément de structuration de l'offre de soins, voire un élément d'accréditation de nos structures.

Une réponse partielle a été apportée par l'INCa au nom du groupe de travail auquel de nombreux orateurs/oratrices d'Avignon ont participé en proposant en janvier 2019 de «nouveaux indicateurs de qualité et de sécurité des soins « pour améliorer le parcours de soins des patientes atteintes de cancer du sein.

Cela nous montre que ce congrès d'Avignon 2018 a été un moment intéressant de partage dans ce vaste champ de la réflexion pour améliorer nos pratiques et que le chemin qui reste à parcourir est encore long. À mon avis, il n'a pas de fin.

En remerciant encore tous ceux et toutes celles qui ont pu nous envoyer leur texte pour être publié dans ce numéro d'Oncologie SFSPM - Avignon 2018, nous vous souhaitons une bonne lecture.

Avignon est dans sa tradition historique, un congrès de la SFSPM tous les dix ans (nous vous attendons en 2028) et reste un lieu de concertation et de réflexion pour le futur. Nous espérons avoir rempli au moins en partie notre objectif de nous faire réfléchir sur l'optimisation du parcours de soins des patientes atteintes de cancer du sein. 